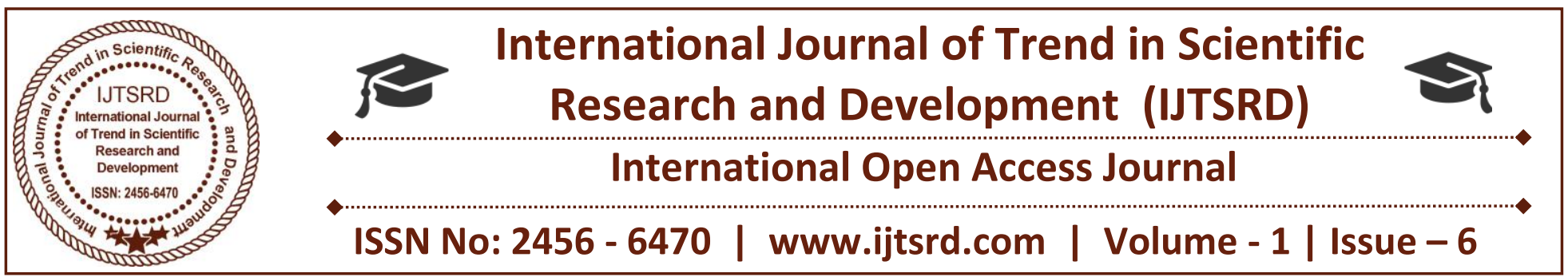

\title{
A Review on Detection of Cracks Present in Composite Cantilever Beam by using Vibration Analysis Technique
}

\author{
Mr. KadamSatish P. \\ P. G. Student, Mechanical Engineering, \\ SVERI'S College of Engineering, \\ Pandharpur, Maharashtra
}

\author{
Dr. Kachare P.S. \\ Project Guide (H.O.D), Mechanical Engineering, \\ SVERI'S College of Engineering, \\ Pandharpur, Maharashtra
}

\section{ABSTRACT}

The aim of this paper is to discuss various techniques used by various researchers for vibration based crack detection in cracked composite structures. In aeronautical, mechanical and civil engineering fields various structural systems are likely to damage and deterioration during their working period. So it is very important to find damage and deterioration in structural member by an effective and reliable methodology. Interest in various damage detection methods has considerably increased in last few decades. A local flexibility is introduced in structural member due to the presence of crack that would affect the vibration response of structure. Due to the presence of crack there is reduction in stiffness of structure and increase in damping of the structure. Since there are changes in physical properties there are reductions in natural frequencies and deviation in mode shapes. Therefore by measuring the vibration parameters it is possible to predict crack depth and crack location in structural member. In this paper effect of various parameters like crack depth, crack location on natural frequency of beam is studied.

Presence of crack in abeam decreases the natural frequency which is more pronounced when the crack is near the fixed support and the crack depth is more.

Keywords: Vibration, Natural frequency, Crack depth, Crack location, Mode Shapes

\section{1) INTRODUCTION:}

The occurrence of defects, cracks and damages may be inevitable in engineering structures in spite of our best efforts. They are formed due to various causes. Some of them are metallurgical defects, generated as a result of improperly controlled manufacturing processes. Some may be environment induced cracks, such as stress corrosion cracking and hydrogen damage. Some may be fatigue cracks that occur under cyclic service load conditions controlled by the limited fatigue strength of materials.

A crack which occurs in a structural element would reduce the local stiffness and weaken the structural strength. Even though all structures are designed to withstand the expected operational loads, they are prone to damages from overloads or repeated fatigue loads leading to cracks. The continued and undetected growth of the crack may finally lead to the collapse of structure, and subsequently to the possible losses of human lives and tremendous revenues. Therefore, the detection of a crack at its early stage of development is important for the safety of the structure and human lives. During the last few decades, focused efforts have been made for the development and improvement of existing and developing nondestructive testing (NDT) methods. The use of various materials, types of structures, construction procedures. etc., have led to the development of different NDI methods. Some methods such as Visual inspection, Ultrasonic, Radiography, Acoustic Emission, 
Magnetic Particle inspection, Dye Penetrant and Eddy Current techniques, are called conventional methods which are well developed and already in use for detecting cracks/flaws in Structures. The other methods such as Vibration Based Inspection, Wave Propagation and Laser techniques are new emerging methods which are undergoing development and not accepted as readily as others. Most of NDT methods could be more or less considered to be applicable to general use; but these methods also have some kinds of limitations, which make them excellent for detecting one kind of damage and useless for detecting others.

As a newer NDT method, the Vibration analysis has been proposed as one of the most promising approaches ${ }^{[1]}$. Vibration analysis, which can be used to detect structural defects such as cracks, of any structure offers an effective, inexpensive and fast means of non-destructive testing. The fundamental idea of vibration analysis is to get information about the soundness of a structure from the measurement of its vibration characteristics; if there is a change in the vibration characteristics (Which may be due to a number causes) of the structure, then such changed characteristics could be due to a decrease of stiffness caused by the presence of cracks.

Vibration analysis, in theory, is generally developed from the investigation of simple structures such as a beam. From vibration theories of beams, one knows that natural frequencies of a beam are dependent on the square root of flexural stiffness of the beam. The local flexural stiffness in a structure would change due to the crack and how much it would change, globally and locally, is dependent on the crack size and location. Given the size and location of the crack, it is possible to calculate the changes of vibration characteristics of the structure, based on the correct crack models and solution methods. Conversely, if the changes of vibration characteristics are measured from vibration response, it is also possible to detect the crack and find its location and size.

Crack detection methods are developed based on crack modeling and experiments. Basically, there are two variations in vibration based crack detection methods: frequency analysis and modal analysis, which need to record sensor data in time domain and then to transform data into frequency domain using Fast Fourier transform procedures. Infrequency analysis, one simple method is to compare the measured frequencies to the predicted frequencies of a cracked structure to determine the presence of the crack. Other methods are often involved in defining some parameters (indicators), which are related to frequency shifts, to determine the crack size and/or location. In modal analysis, many crack indicators have been developed based on frequencies and mode shapes, which include modal assurance criteria (MAC), mode shape curvature/strain, modal sensitivity and frequency transfer function.

In this paper efforts have been made to present various cost effective reliable analytical numerical and experimental techniques developed by various researchers for vibration analysis of cracked beams. In this paper the effect of various parameters like crack size, crack location, of beam on modal parameters subjected to vibration of a damaged beam also have been reviewed.

\section{2) LITERATURE REVIEW:}

Murat Kisa, ${ }^{[1]}$ has presented new methods for numerical modeling of the free vibration of cantilever composite beam having multiple open and nonpropagating cracks. This study investigated effects of cracks on dynamic characteristics of cantilever composite beam, made of graphite fibre-reinforced polyamide. The finite element and the component mode synthesis methods are used to model the problem. The presented method is adequate for the vibration analysis of cracked cantilever composite beam, and by using drop in natural frequency and change in mode shapes, the presence and nature of cracks in structure can be detected.

Kaushar H. Barad, ${ }^{[2]}$ has presented detection of crack in cantilever beam by using natural frequency. The method presented by him to detect crack location and size is fast and efficient. Crack with larger crack depth ratio $(\mathrm{a} / \mathrm{h})$ imparts greater reduction in natural frequency than that of smaller crack depth ratio. Accuracy of results can be improved with increasing the crack depth. Crack present near to the fixed end imparts greater reduction in natural frequency than that to present at away from the fixed end. Experimental setup used in this paper is as shown below 


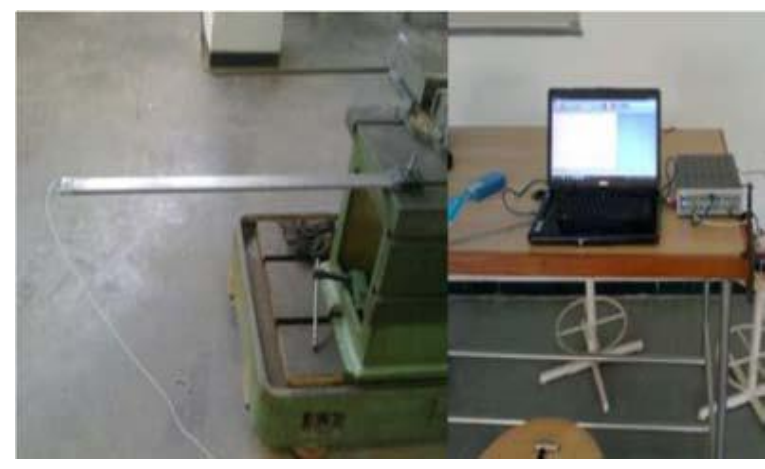

Fig: Experimental setup for crack detection of cantilever beam with single crack $^{[2]}$

K.Oruganti, ${ }^{[3]}$ has presented study of testing carbon/epoxy composite beam with various embedded delaminations with mechanical actuator and scanning laser vibrometer (SLV) as a sensor for recording the frequency response and analyzing the acquired signatures based on displacement and curvature mode shapes. He also includes Finite Element Analysis (FEA) and C-scan techniques used on the composite beams to validate the results. Experimental setup used in their work is as below

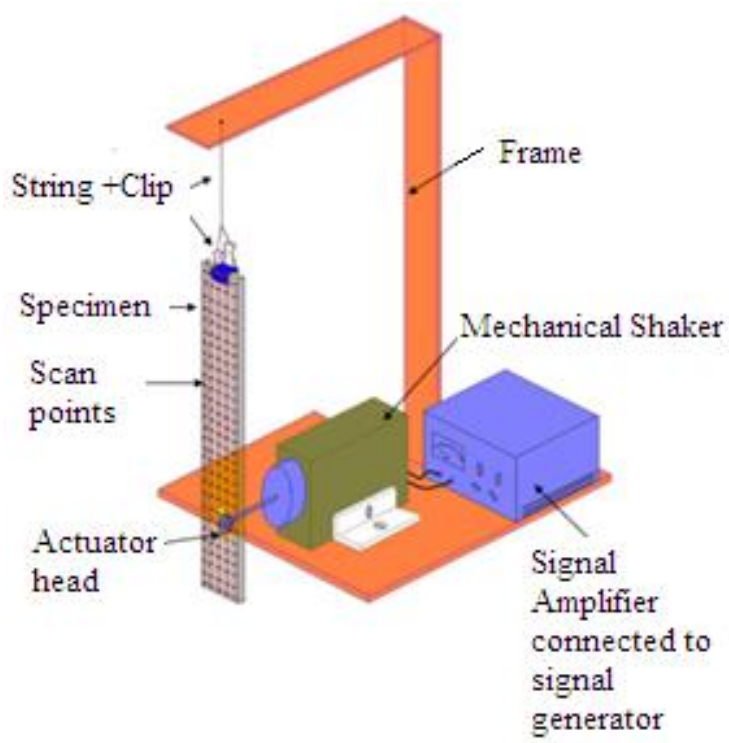

\section{Fig: Experimental setup for analysis of damage in composites $^{[3]}$}

The damage within the Carbon/Epoxy beams could be identified successfully by both the displacement mode shape analysis as well as curvature mode shape analysis. The displacement mode shape provides a global snapshot through the frequency domain range, is a valid method for a globalized technique of damage identification. The curvature mode shape being the double derivative of the displacement mode shape is more of a localized technique. The size and location of the delamination in one place was successfully identified by both these techniques and validated using the $\mathrm{C}$-scan results.

Free and forced vibration analysis of cracked beam was performed by SadettinOrhan, ${ }^{[4]}$ in order to identify the crack in a cantilever beam. Single- and two-edge cracks were evaluated. The study results suggest that free vibration analysis provides suitable information for the detection of single and two cracks, whereas forced vibration can detect only the single crack condition. However, dynamic response of the forced vibration better describes changes in crack depth and location than the free vibration in which the difference between natural frequencies corresponding to a change in crack depth and location only is a minor effect.

The fault detection of multi cracked slender Euler Bernoulli beams through the knowledge of changes in the natural frequencies and their measurements has been presented by P.K.Jena ${ }^{[5]}$. The method is based on the approach of modeling a crack by rotational spring. The spring model of crack is applied to establish the frequency equation based on the dynamic stiffness of multiple cracked beams. According to him the mode shapes and natural frequencies of the cracked elastic structures are strongly influenced by the crack location and its intensity. The significant changes in mode shapes are observed at the vicinity of crack location. The positions of the cracks in relation to each other affect significantly the changes in the frequencies of the natural vibrations in the case of an equal relative depth of the cracks. In the case of two cracks of different depths, the larger crack has the most significant effect on the natural vibration frequencies.

A review on the vibration analysis for a damage occurrence of a cantilever beam has been presented by Z.A. Jassim ${ }^{[6]}$. He suggested that based on the changes in dynamic properties such as the stiffness which could lead to changes in:

1. The mode shape

2. Reduction in frequencies

3. Increase in damping,

4. In addition, the location and the magnitude of the crack can be determined

The vibration analysis for cantilever beam with crack were illustrated analytically, theoretically and experimentally; with the beams having various properties and dimensions. 
AbdeldjebarRabia [7] has presented vibration experimental method necessary for the determination of mechanical characteristics of the studied structure. This technique allowed to determine the two Young modulus E1, E2 by the consideration of longitudinal vibrations according to the direction $\mathrm{x}$ and transversal vibrations according to the direction $y$.

Cost effective and reliable damage detection is critical for the utilization of composite materials. Seth S. Kessler, ${ }^{[8]}$ has presented part of an experimental and analytical survey for the detection of damage in composite materials. The potential role of the frequency response of composite structure in SHM system was investigated in this paper. A finite element model was built to numerically explore the effects of various types of damage on the normal modes of test specimens, and analogous experiments were performed using a scanning laser vibrometer and impedance meter to verify these results. Good correlation was found between the model and the experimental results for low frequencies. In both numerical model and the experimental results there was strong correspondence between the extent of damage (or local stiffness loss) and reduction in natural frequency, which again was mostly measurable at lower frequencies.

The effect of the impact failure on the natural frequency and damping ratio has presented by ZekiKiral ${ }^{[9]}$. The fundamental vibration frequency of the beam was measured experimentally using noncontact technique for different failure locations and damping ratio is calculated from the envelope of the free vibration response. Based on the results obtained in this study, the following conclusions can be drawn.

1. The experimental results show that the variation in the natural frequency value versus severity and location of failure is not steady and measurable for the considered real failure case.

2. The numerical results show that the presence of damage brings an imperceptible reduction in natural frequencies of a cantilever beam. The decrease in natural frequencies disappears as the failure moves away from clamped edge.

3. The damping ratio increases at measurable level as the damage severity increases and damping ratio is more sensitive to damage when the location of damage is near to clamped edge.

JeslinThalapil, ${ }^{[10]}$ has presented method for detection of longitudinal cracks in long and short beams using changes in natural frequencies. The accuracy of this method for prediction of natural frequencies is illustrated by case studies involving both long and short beams with known longitudinal crack details. The natural frequencies show good agreement with the ANSYS results. The method of prediction of crack parameters for a longitudinal axial internal crack has been verified for both long cantilever and simply supported beams. The range of crack size considered is 5-45\%. In case of long cantilever beams, the maximum error in prediction of crack location is $4 \%$ and the error in prediction of crack size is $2 \%$. The same errors in the case of simply supported beams are $2 \%$ and $3 \%$ respectively.

The method has been used to predict the parameters of a longitudinal edge crack offset with respect to the axis in both long and short cantilever beams. The maximum error in prediction of depth from the top edge is $7 \%$ and $4 \%$ in crack size for long beams. The same errors in short beams are 5\% and $4 \%$ respectively.

Jialai Wang, PizhongQiao ${ }^{[11]}$ has presented a new damage detection technique using irregularity profile of a structural mode shape. The mode-shape of a cracked beam is first obtained analytically by using a general function. Its irregularity profile is then extracted from the mode shape by a numerical filter. The location and size of the crack in the beam can be determined by the peak value appearing on the irregularity profile. Two types of numerical filters, i.e., triangular and Gaussian, are examined. It has been found that the former filter is more effective in damage detection than the latter one. Numerical simulations suggest that the irregularity-based method requires a relatively low measurement resolution. Noise stress tests are carried out to demonstrate the effectiveness and robustness of this method under the influence of noise. As a validation, the proposed method is applied to detect crack damage in an Eglass/epoxy laminated composite beam. The successful detection of the crack in the composite beam demonstrates that the irregularity-based method is capable of assessing both the location and size of the crack and can be used efficiently and effectively in damage identification and health monitoring of beam-type structures.

In this paper, the fundamental issues (e.g., single crack vs. multiple cracks, crack size, Gaussian vs. triangular filters, resolution requirements, and noise effect) of irregularity-based damage detection method are addressed. It is shown that the proposed method can successfully detect not only a single crack, but 
also multiple cracks in beams. The location and size of a crack in beams are determined by a peak value at the location of the damage on the irregularity profile of mode shape. Compared with existing damage detection methods such as wavelet transform, the proposed method has a much easier calculation scheme and more straight forward physical meaning.

An experimental damage identification procedure based on structural dynamic responses and using smart sensors is conducted by Wahyu Lestari et al. ${ }^{[12]}$ In this study, a damage detection and structural health monitoring procedure based on curvature mode shapes and using piezoelectric smart sensors is developed. It indicated that the proposed technique can be effectively and appropriately applied to full scale composite structures (e.g., large FRP honeycomb sandwich structures) suitable for civil infrastructure. The locations of the damage for both damage configurations (i.e., the core-face plate debonding and core crushing, respectively) were identified properly using the curvature damage factor and damage index method, while the magnitude of the damage was evaluated through the stiffness loss. The damage detection technique proposed in this study can be implemented in inherent damage identification and health monitoring of large civil composite structures.

Although the implementation of the method is quite simple, a great care needs to be taken while generating experimental data. Number of measurement points will define the maximum number of mode shapes generated. The larger number of measurement points is, the more mode shapes can be generated. However, using the lower curvature modes yields better results than using the higher modes, as at higher modes the nodal curvature points of damaged modes shifting from the original position of undamaged modes.

A combined analytical and experimental study of flexural-torsional buckling of pultruded fiberreinforced plastic (FRP) composite cantilever I-beams has presented by PizhongQiao et al. ${ }^{[13]}$ In this paper, a combined analytical and experimental study is presented to study the flexural-torsional buckling behavior of pultruded FRP composite cantilever Ibeams. The total potential energy based on nonlinear plate theory is derived, and shear effects and beam bending-twisting coupling are accounted for in the analysis. Three different types of buckling mode shape functions, namely transcendental function, polynomial function, and half simply supported beam function, which all satisfy the cantilever beam boundary conditions, are used to obtain the analytical solutions and explicit prediction formulas. An experimental study of four different geometries of FRP cantilever I-beams is performed, and the critical flexural-torsional buckling loads for different span lengths are obtained. A good agreement among the proposed analytical solutions, experimental testing, and FEM is obtained. The study on effect of buckling mode shape functions indicate that the approximations by exact transcendental function and polynomial function compare well with FEM results and may be more applicable for the buckling modeling of cantilever beam configuration. A parametric study on the effects of load location through the height of the cross-section, fiber orientation and fiber volume fraction on buckling behavior is also presented. The explicit and experimentally validated analytical formulas for the flexural-torsional buckling prediction can be effectively used to design and characterize the buckling behavior of FRP structural shapes.

ErfanAsnaashari,JyotiK.Sinha, ${ }^{[14]}$ has developed method for development of residual operational deflection shape for crack detection in structure.Excitation of cracked structure at frequency always generates higher harmonic components of the exciting frequency due to breathing of crack. In this paper, the deflection of cracked structures at the exciting frequency and second harmonic component is mapped by new method base on operational deflection shape (ODS) for the purpose of crack detection. While ODS is helpful in understanding dynamic behavior of structures and machines, it is not always possible to determine the location of cracks in structure based on the ODS itself. Therefore new concept called residual ODS (R- ODS) has been defined for crack detection in beam like structure.

Zhichun Yang, et, al. ${ }^{[15]}$ has proposed new concept of inner product vector (IPV) and it is proved that under white noise excitation the IPV of the structure is dependent on the modal parameters of the structure. Using the IPV as a damage index, the corresponding detection method is presented. The damage detection examples for the delamination damage of a composite beam show that

1. The IPV-based damage detection method can locate the delamination of the composite beam correctly, even if the measurement noise of up to $10 \%$ of the root mean square value of the response signal is considered. 
2. In the IPV-based damage detection method, only the vibration responses excited by random excitation are required, and it is clear that in practice, the finite element model of the structure is not required. As the algorithm is rather simple, the IPV-based damage detection method can be used as a real-time or online damage detection method.

Nevertheless, like many of the existing damage detection methods, the IPV-based damage detection method cannot be used to locate the damage if the influence of the damage on the structures dynamics is too slight when the measurement noise is too large. Though almost all the damage cases were detected by the minimum of the invariable threshold and variable threshold, how to choose a more appropriate threshold for damage detection is a critical issue for such a method. Meanwhile, the other shortage of the IPVbased damage detection method is the cost of sensors which means that the sensor should be located close to the damage.

An attempt has been made by Ramdas C. et al. ${ }^{[16]}$ to combine damage detection features of ultrasonic Lamb wave (TOF and amplitude ratio) with vibration based technique features (first and second natural frequencies) in an ANN environment with a probability of detection of $89 \%$. It was studied that when damage features of more than one technique are combined, the damage could be identified more effectively than using damage features of each technique individually. If techniques like Lamb wave and vibration are employed individually for sizing of transverse cracks, Lamb wave technique fails when the damage zone is close to fixed boundary and vibration technique fails when the damage zone is close to free edge. By combining these two techniques the domain of damage detection is enhanced, which covers almost the whole sample. This study showed that ANN could form an efficient tool in detecting the damage location and depth.

\section{3) MATERIALSAND METHODS:}

Vibration analysis of nonmetallic material is followed by authors with different methods such as theoretical, experimental, finite element method, Artificial Neural Networks. Vibration analysis of metallic material is done by theoretical, wavelet analysis, finite element analysis, MATLAB methods. Study of Glass/polyester, fibre glass, E-glass fibre epoxy resin is done by using numerical model analysis, compliance matrix, and numerical, experimental methods.

\section{4) DISCUSSION:}

Earlier, using the different non-destructive evaluation techniques cracked vibrating structures are effectively analyzed by various researchers. Some researcher suggested that changes in dynamic characteristics can be used as an information source for detecting of vibrating beam or structure in presence of crack. While working on different structures, researchers has studied the effect of crack location, crack depth, crack inclination on natural frequency of a cracked beam subjected to vibration. For determination of crack location and crack depth transfer matrix method uses the input data of changes in mode shapes and natural frequencies. The physical dimensions, boundary conditions and the material properties of the structure play important role for the determination of its dynamic response. The position, depth ratio, orientation and number of cracks are greatly influence the dynamic response of the structure.

For diagnosis of crack in a vibrating beam structure many researchers have worked on the application of artificial neural network and fuzzy logic concept. Some have worked on the application of continuous wavelet analysis for detecting of crack in vibrating beam. Concept of fracture mechanics, stress intensity factor and knowledge of strain energy release rate has been used for analysis of crack detection.

\section{5) CONCLUSION:}

It has been observed that the changes in natural frequencies are the important parameter that determine crack size and crack location respectively. If the crack location is kept constant then crack depth and natural frequency are inversely proportional to each other. Natural frequency decreases with increase in crack location from the cantilever when crack depth is keeping constant. It is obvious that the vibration behavior of the beams is very sensitive to the crack location, crack depth and mode number. The direction of crack does not affect the natural frequency. Crack with larger crack to depth ratio $(\mathrm{a} / \mathrm{h})$ imparts greater reductions in natural frequency than that of the smaller. Researchers are presently focusing on using the concept of fuzzy logic as an effective tool for crack analysis of structures. 


\section{REFERENCES:}

[1] Murat Kisa,"Free Vibration Analysis of a Cantilever Composite Beam with Multiple Cracks.", Composite Science and Technology.2004, pp13911402.

[2] Kaushar H. Borad et al, "Crack Detection in Cantilever Beam by Frequency Based Method", Procedia Engineering, 2013, pp770-775.

[3] K. Oruganti et al," Vibration-Based Analysis of Damage in Composites" Materials Forum Volume33, 2009, pp496-504.

[4] SadettinOrhan, in "Analysis of free and forced vibration of cracked cantilever beam", NDT\&E International 40(2007) 443-450

[5] P.K. Jena,D.N. Thatoi,J. Nanda,D.R.K. Parhi], in "Effect of damage parameters on vibration signatures of a cantilever beam", Procedia Engineering 38 (2012) 3318-3330

[6] Z. A. Jassim et al,"A Review on the Vibration Analysis for a Damage Occurance of a Cantilever Beam” Engineering Failure Analysis,2013,pp442-461.

[7] AbdeldjebarRabia et al,"Dynamic

Characterization by experimental analysis of composite beam "Energy Procedia 36 (2013) 808-814

[8] Seth S. Kessler et al,'Damage detection in composite materials using frequency response method" Composites: Part B 33 (2002) 87-95

[9] ZekiKiral et al,'Effect of impact failure on damping characteristics of beam like composite structure.” Composites: Part B 43 (2012) 3053-3060

[10] JeslinThalapil et al," Detection of Longitudinal Cracks in Long and Short Beams Using Changes in Natural Frequencies" International Journal of Mechanical Sciences, 2014, pp38-47.

[11] Jialai Wang, PizhongQiao "On Irregularitybased Damage Detection Method for Cracked Beams" Inernational Journal of Solids and Structures, 2008, pp688-704.

[12] Wahyu Lestari, PizhongQiao, "Damage Detection of Fiber Reinforced Polymer Honeycomb Sandwich Beams" Composite Structures. 2005, pp365-373.

[13] PizhongQiao, GuipingZou,JulioF.Davalos, "Flexural-torsional Buckling of Fiber Reinforced Plastic Composite Cantilever I Beams" Composite Structures, 2003, pp205-217.
[14] ErfanAsnaashari et al,"Crack Detection in Structures Using Deviation from Normal Distribution of Measured Vibration Responses" Journal of Sound and Vibration,2014,pp4139-4151.

[15] Zhichun Yang et al,'Damage Detection in Composite Structures Using Vibration Response Under Stochastic Excitation" Journal of Sound and Vibration, 2009, pp755-768.

[16] Ramdas C. Krishnan Balasubramaniam et al'Detection of Transverse Cracks in a Composite Beam using Combined Features of Lumb Wave and Vibration Techniques in ANN Environment" International Journal on Smart Sensing and Intelligent Systems, Vol.1, 2008, pp970-984. 